\title{
El lento retorno de las políticas industriales en América Latina y el Caribe
}

\author{
Wilson Peres
}

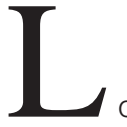

esencial de una política para acelerar el crecimiento económico es combinar la acumulación de conocimiento y la diversificación de la estructura productiva. Estas dos dimensiones son el eje de este artículo, que examina experiencias de política industrial durante el período de industrialización mediante la sustitución de importaciones; pone de relieve las políticas que se aplican actualmente en la región y elabora una tipología de sus estrategias; presenta las principales líneas de acción e instrumentos de política, y analiza cuestiones relativas a la evaluación de la puesta en práctica y el impacto de las políticas aplicadas. La tesis es que, si bien se ha avanzado en la capacidad de formular políticas, aún son necesarios significativos esfuerzos para mejorar su implementación y desarrollar metodologías que permitan evaluar su impacto adecuadamente. De esa evaluación se desprenderá cuál ha de ser la aceptación que las políticas industriales tendrán en América Latina y el Caribe.

Jefe de la Unidad de Desarrollo Industrial y Tecnológico,

División de Desarrollo Productivo

y Empresarial de la CEPAL

œwilson.peres@cepal.org 
I

\section{Introducción}

Este trabajo parte de la hipótesis de que, para potenciar sus procesos de desarrollo, los países de América Latina y el Caribe deben poner en práctica políticas que apunten a crear nuevos sectores productivos o a modernizar los sectores maduros, respetando las restricciones dadas por el tamaño, el grado de desarrollo y la estructura productiva de las distintas economías nacionales. ${ }^{1}$ La diversificación de la estructura productiva, mejorando la mezcla de productos y el vector de especialización internacional, es un factor determinante de la disminución de la brecha de productividad entre estos países y los de la frontera tecnológica internacional y, por lo tanto, de la aceleración del crecimiento de la productividad agregada en economías abiertas. Tal diversificación permite mejores eslabonamientos internos, lo que fortalece el impacto positivo del crecimiento económico sobre la productividad agregada (Cimoli, Correa y Primi, 2003).

Las políticas dirigidas al fortalecimiento sectorial pueden aumentar la densidad y complejidad de la estructura productiva de un país, las que están positivamente correlacionadas con la estabilidad de sus tasas de crecimiento, así como con la rapidez y flexibilidad de su respuesta a shocks externos (Castaldi, 2003). La complejidad productiva actúa creando contrapesos internos a la transmisión de los shocks, generando así estabilizadores automáticos. Tanto la especialización con mayor contenido de conocimiento como la diversificación permiten aprovechar a cabalidad los rendimientos crecientes a escala implícitos en el progreso técnico, lo que conduce directamente a procesos virtuosos de causalidad acumulativa (Young, 1928; Stigler, 1951; Kaldor, 1966).

\footnotetext{
${ }^{1}$ Este trabajo se realizó en virtud del proyecto "Fomento al desarrollo productivo", en el marco del Programa CEPAL/GTZ de "Modernización del Estado, desarrollo productivo y uso sostenible de recursos naturales", y se presentó en la Segunda Reunión del grupo de trabajo sobre política industrial (Río de Janeiro, marzo del 2005) de la Iniciativa para el Diálogo de Políticas. El autor agradece en particular las observaciones de Antonio Barros de Castro (comentarista de la presentación), Alice Amsden, Mario Cimoli, Giovanni Dosi, Bernardo Kosacoff, Yevgeny Kuznetsov, Richard Nelson, Gabriel Palma y Gabriel Porcile. Algunas partes de este trabajo fueron publicadas en CEPAL (2004a, cap. 8).
}

En síntesis, el meollo de una política para acelerar el crecimiento de la productividad en el largo plazo reside en combinar la acumulación de conocimiento y la diversificación de la estructura productiva. $\mathrm{La}$ primera genera las posibilidades; la segunda las concreta. Ambas dimensiones están presentes en este trabajo, que se organiza en cinco secciones. Luego de esta sección introductoria, en la que se pasa revista a las grandes líneas de las experiencias en política industrial durante el período de industrialización sustitutiva de las importaciones, en la sección II se examinan las prácticas de política en curso en la región y se elabora una tipología de las estrategias que las sustentan. En la sección III se presentan las principales líneas de acción e instrumentos de política, mientras que en la sección IV se analizan temas vinculados a la implementación y la evaluación del impacto de las políticas utilizadas. Por último, en la sección V se presentan algunas conclusiones.

Las políticas dirigidas a crear nuevos sectores fueron el centro de la política industrial en el modelo de sustitución de importaciones. ${ }^{2}$ Incluso, en la actualidad, se suele definir esas políticas como las que buscan modificar el vector de producción de bienes y servicios (Chang, 1994; Melo, 2001), lo que necesariamente implica la creación de nuevas actividades. El objetivo de esas políticas era completar el tejido industrial de los países, aprovechando los efectos del crecimiento de la demanda interna, particularmente de inversión, los que de otro modo se traducirían en aumento de importaciones, con el consiguiente impacto negativo sobre la restricción externa. Durante la década de 1970 se fortaleció la percepción de que los efectos de la inversión se dividían en dos; por un lado, la instalación de capacidad productiva, con impactos positivos sobre la oferta agregada; por otro, la demanda derivada de bienes de capital que, por insuficiencia de la oferta interna adecuada, se traducía en demanda de importaciones, perdiéndose así los efectos de arrastre

\footnotetext{
${ }^{2}$ En este documento, los términos industria e industrial se usan en sentido amplio, es decir, no incluyen sólo a la industria manufacturera, sino también a sectores no manufactureros, como el agrícola y el minero. En ese sentido, se los usa como sinónimos de sector y sectorial, o del término industry en inglés.
} 
sobre el resto de la estructura productiva. En ese momento, los conceptos de política industrial, política manufacturera y política de fomento a la producción de bienes de capital estaban estrechamente vinculados.

La política industrial tuvo como principal instrumento una combinación de protección comercial, promoción de inversiones directas - a menudo estatales o extranjeras- y financiamiento otorgado por los bancos nacionales de fomento. Los ejemplos más notorios en la década de 1970, antes del quiebre que significó la crisis de la deuda externa, fueron el II Plan Nacional de Desarrollo en Brasil y el Programa Nacional de Fomento Industrial 1979-1982 en México, que estuvo en vigencia durante el auge que acompañó la expansión de la plataforma de exportación de petróleo.

Las políticas industriales ordenaban la expansión de la oferta interna y concentraban los esfuerzos de planificación o programación a nivel de la estructura productiva. Tres factores vinculados entre sí fortalecían ese papel ordenador: i) los mecanismos de fomento del sector público se organizaban a nivel sectorial e incluso subsectorial; ${ }^{3}$ ii) los intereses privados se organizaban también en cámaras o asociaciones sectoriales, que eran las principales defensoras de la estructura de protección comercial, y iii) las negociaciones comerciales internacionales (por ejemplo, en el marco de la Asociación Latinoamericana de Integración (ALADI), el Mercado Común Centroamericano (MCCA), la Comunidad del Caribe (CARICOM) o el Pacto Andino) se concretaban en listas negativas o positivas, de preferencias sectoriales. Las políticas industriales estuvieron focalizadas en los sectores agropecuario y manufacturero, aunque el peso de este último fue tal que, como se indicó antes, se tendió a confundir los conceptos de política sectorial y de política para la industria manufacturera.

Tras haber ocupado esa posición central, las políticas orientadas al sector manufacturero fueron perdiendo legitimidad a lo largo de la década de 1980, llegando a quedar prácticamente excluidas de la visión del nuevo modelo económico implantado con las reformas estructurales, al menos en su formulación más estricta. La pérdida de aceptación de este tipo de políticas tuvo diversas causas. Las principales fueron: la privatización o cierre de empresas públicas que invertían directamente en nuevos sectores, puesto que, se-

\footnotetext{
${ }^{3}$ Por ejemplo, ministerios de industria, agricultura, minería y otros y, dentro de ellos, direcciones generales de alimentos, metalmecánica, química, bienes de capital, etc.
}

gún la nueva visión del Estado, este solo debía desempeñar un papel subsidiario en la dinámica económica; la necesidad de equilibrar las finanzas públicas mediante la eliminación de subsidios, en particular los fiscales y los componentes de subsidio de las operaciones de crédito, y la percepción, algunas veces controvertible, de que numerosas inversiones habían implicado mala planeación, gestión deficiente de proyectos, corrupción e incluso proyectos inútiles como los llamados "elefantes blancos". Esta pérdida de legitimidad no se dio en todas partes del mundo. Así, por ejemplo, en varios países del este y sudeste de Asia, las políticas activas con alcance sectorial e incluso focalizadas a nivel de empresa se mantuvieron vigentes hasta bien entrado el decenio de 1990, pero perdieron fuerza a medida que esos países se fueron incorporando paulatinamente, aunque con diferentes ritmos, a la corriente de libre mercado y al nuevo régimen internacional de comercio. ${ }^{4}$

Más allá de los argumentos económicos contra la política industrial, la oposición al nuevo modelo económico de los agentes políticos que favorecían esa política llevó a que se consolidara el estereotipo "desarrollistas versus neoliberales”. Esa oposición a las reformas estructurales fue combatida por los agentes que apoyaban las reformas mediante la acentuación de un discurso que culpaba a las políticas industriales sectoriales de distorsionar la asignación de recursos y causar los desequilibrios fiscales que estaban detrás de los procesos inflacionarios. Esta posición de crítica a la política industrial fue compartida por un número creciente de gobiernos en la región. ${ }^{5}$ Sin embargo, esa visión extrema no siempre se condijo con los hechos; incluso gobiernos fuertemente reformadores, como los de Menem en Argentina, Collor de Melo en Brasil y Salinas de Gortari en México, mantuvieron ciertas políticas sectoriales, en particular para la industria automotriz.

\footnotetext{
${ }^{4}$ El debate sobre el efecto de las políticas industriales en Asia es muy amplio y aún no ha concluido, aunque la crisis de 1997 llevó a que, en el caso asiático, el foco de atención se desplazara de las políticas industriales a otros temas. Véase posiciones favorables a tales políticas en Amsden (1989), Rodrik (1995) y Wade (1990), y posiciones contrarias en Banco Mundial (1993), Krugman (1994) y, más recientemente, Noland y Pack (2002). Por otra parte, las políticas agrícolas de los países industrializados muestran que las políticas sectoriales están lejos de ser una peculiaridad exhibida por algunos países subdesarrollados en el pasado.

5 A comienzos del decenio de 1990, era frecuente escuchar de altos funcionarios a cargo de la política macroeconómica el apotegma "la mejor política industrial es no hacer política industrial". Pese a su simplicidad, esa frase reflejaba adecuadamente su postura frente al tema.
} 


\section{II}

\section{Práctica y estrategia de las políticas industriales}

\section{La experiencia de la región}

Gran parte de la experiencia actual de la región en materia de política industrial está incluida en la expresión "políticas de competitividad" (Peres, 1997). ${ }^{6}$ Las políticas vigentes en la región se pueden ordenar en cuatro grandes grupos:

i) Políticas que continúan las que se desarrollaron en el período de la industrialización por sustitución de importaciones y que buscan expandir y profundizar un sector particular, integrando nuevos segmentos y aplicando alguna combinación de protección comercial e incentivos fiscales y financieros. Ejemplos notorios de estas políticas son los regímenes para la industria automotriz en el Mercosur, tendientes a ordenar y expandir las inversiones de empresas productoras de automóviles y de partes (CEPAL, 2004b, recuadros III.3 y III.4). En muchos países de la región ha habido apoyos esporádicos a sectores sensibles (de débil competitividad) como los de textiles, prendas de vestir, calzado, productos electrónicos y juguetes, y también a numerosos productos agrícolas y actividades mineras que varían según los países; estos apoyos han sido mucho más estables que los incentivos a las actividades manufactureras. Incluso en el caso de sectores con ventajas comparativas reveladas, como algunos segmentos importantes del sector agrícola, con frecuencia ha habido que establecer esquemas para ayudarlos a enfrentar crisis de corto plazo $^{7}$ o desafíos

\footnotetext{
${ }^{6}$ Las consideraciones sobre el impacto de las reformas económicas y de la política macroeconómica sobre la dinámica industrial escapan al alcance de este trabajo. Sin embargo, hay que resaltar que muchas veces reformas como la apertura comercial o las privatizaciones, y las medidas de política monetaria y cambiaria, han influido con fuerza sobre esa dinámica, lo que ha llevado a considerarlas verdaderas "políticas industriales implícitas". Tales políticas implícitas muchas veces fueron decididas sin un conocimiento adecuado de la microeconomía de la región, es decir, de las dinámicas específicas de sus empresas y mercados (véase Stallings y Peres, 2000).

${ }^{7}$ Por ejemplo, las exenciones tributarias a los productores de carne en momentos de crisis de fiebre aftosa en Uruguay, señaladas por Scarone (2003). Por su parte, datos de 2003 sobre Brasil muestran apoyos significativos, entre otros, al sector de energía eléctrica (mil
}

de más largo plazo debidos a pérdidas relativas de competitividad. $^{8}$

En el sector agrícola, son varios los países (entre ellos los centroamericanos, Brasil, Colombia y la República Dominicana) en que se mantiene la intervención directa en los mercados de granos básicos (trigo, maíz, arroz). Sin embargo, cada vez más las intervenciones directas en los mercados - por ejemplo, mediante precios de garantía - y el crédito subsidiado son sustituidos tanto por programas destinados a los pequeños productores, que son los más afectados por la apertura comercial, como por instrumentos horizontales: entre otros, gastos en programas de sanidad animal y vegetal, riego y titulación de tierras (FAO, 2001 y CEPAL, 2003). Asimismo, se da importancia cada vez mayor a programas de alcance territorial o local, como los de incentivos fiscales en regiones pobres o los de desarrollo rural integral, así llamados porque combinan inversión en infraestructura con capacitación y asistencia técnica en áreas rurales relativamente desfavorecidas.

ii) Políticas focalizadas en sectores, que evolucionaron hasta transformarse en políticas con impacto sobre el conjunto del sistema económico. Este es el caso de aquellas para la industria electrónica e informática, que comenzaron como políticas de sustitución de importaciones de equipos (hardware), luego se dedicaron a apoyar el desarrollo de un intangible (software) y han sido integradas en las estrategias para el desarrollo de las tecnologías de la información y las comunicaciones (TIC) e incluso para dar nacimiento a "sociedades de la información" en América Latina y

millones de dólares en líneas de crédito del Banco Nacional de Desenvolvimento Econômico y Social, BNDES, para capitalizar 24 empresas de distribución); al sector de informática (500 millones de dólares en reducción del impuesto sobre productos industrializados, IPI), al de automóviles (120 millones de dólares en reducción del IPI a vehículos pequeños y medianos) y al de electrodomésticos (70 millones de dólares en líneas de crédito). Véase Balbi (2003).

${ }^{8}$ Como sucede con el turismo "sol y playa" en parte importante del Caribe de habla inglesa (Hendrickson, 2003), o en México con la reconversión productiva en busca de cultivos con mayor potencial, valor agregado y oportunidades de mercado, en tanto objetivo de la Alianza para el Campo (Villagómez, 2003). 
el Caribe. ${ }^{9}$ La presencia generalizada de economías de alcance y de red, así como la complementariedad entre actividades, lleva a que estas políticas deban ser encaradas transversalmente, sobrepasando límites sectoriales o institucionales que, por otra parte, tienden a ser cada vez más borrosos.

iii) Políticas centradas en sectores con elevada concentración, derivadas de economías de escala y de red (energía eléctrica, telecomunicaciones, petróleo y gas natural). En estos sectores las políticas, en casi todos los casos decididas después de procesos de privatización, se han orientado a desarrollar marcos eficientes de regulación, lo que ha significado crear y fortalecer agencias reguladoras, adecuar el marco normativo y esforzarse por articular la expansión de las inversiones en estos sectores con la oferta de los proveedores internos, articulación cuya intensidad varía de un país a otro. ${ }^{10}$ En Brasil se ha llegado incluso a establecer "fondos tecnológicos" para apoyar programas de desarrollo científico y tecnológico en cada uno de los sectores en cuestión, con recursos provenientes de las regalías que pagan las empresas.

iv) Políticas de apoyo a los conglomerados productivos (clusters), en particular los integrados por empresas pequeñas y medianas, o por muchas empresas pequeñas y medianas bajo el liderazgo de grandes empresas. Este enfoque ha tenido creciente aceptación en los países andinos y centroamericanos y, al igual que otras políticas industriales, ha buscado acrecentar la competitividad de sectores existentes más que crear nuevas actividades. En países como México y Brasil se han aplicado a nivel subnacional vigorosas medidas para fomentar esos conglomerados. Así lo ilustra en el caso de México el apoyo al sector del calzado en Guanajuato o de la electrónica en Jalisco (Unger, 2003; Dussel Peters, 1999), y en el caso de Brasil las acciones del Servicio Brasileño de Apoyo a las Micro y Pequeñas Empresas (SEBRAE) a lo largo de todo el país, en el marco del proyecto de desarrollo de "arreglos productivos locales" (APL). ${ }^{11}$ Este tipo de política dis-

\footnotetext{
${ }^{9}$ Véase un planteamiento general sobre el tema en CEPAL (2005); en cuanto a análisis nacionales, véase Bonelli y Motta Veiga (2003) para Brasil, Scarone (2003) para Uruguay y Henry (2003) para el Caribe.

${ }^{10}$ Véase Sergeant, Racha y James (2003) para Trinidad y Tabago. ${ }^{11}$ En portugués, arranjos produtivos locais. Las APL se caracterizan por abarcar un número significativo de empresas que actúan en torno a una actividad productiva predominante en un territorio y que comparten formas de cooperación y mecanismos de gobernanza. Las acciones de apoyo a las APL se concretan a nivel local, lo que es consistente con la experiencia brasileña de políticas estaduales
}

fruta de gran legitimidad, incluso entre los organismos financieros internacionales, lo que ha facilitado su aceptación por los gobiernos e incluso ha llevado a que se califique de "apoyo a conglomerados productivos" a medidas en favor de actividades que, en sentido estricto, no tienen alcance ni de cadena productiva ni de conglomeración geográfica. ${ }^{12}$

\section{Una tipología de estrategias nacionales}

Después de que resurgiera el interés por políticas activas de alcance microeconómico y sectorial a mediados del decenio de 1990, tomaron forma tres maneras de enfocar las políticas de competitividad. En algunos países, fundamentalmente Brasil, México y los del Caribe de habla inglesa, se elaboraron documentos de política orientados específicamente al sector industrial y a su vinculación con el desarrollo tecnológico y la inserción internacional. ${ }^{13}$ Más que planes o programas industriales en un sentido estricto, estos documentos fueron agendas de trabajo entre el gobierno y el sector privado, lo que llevó a que sus críticos los acusaran de ser "programas sin metas" e incluso "sin recursos".

En los países andinos y centroamericanos predominaron enfoques tendientes a aumentar la competitividad del conjunto de la economía, sin dar un papel privilegiado al sector industrial, y se formularon estrategias nacionales de competitividad basadas en la metodología del análisis de grupos (clusters), que recibieron distintos nombres, entre otros, los de aglomeraciones industriales, arreglos productivos y conglomerados productivos. ${ }^{14}$ Esta última denominación es la

con un fuerte componente sectorial. Ejemplos de estas políticas son los programas de apoyo a la industria automotriz (mediante subsidios e incluso aportes de capital de algunos gobiernos estaduales), la industria electroelectrónica e informática, la de textiles, la de prendas de vestir y la de calzado. Véase Bonelli y Motta Veiga (2003).

12 Respecto a los acuerdos sectoriales en Colombia, véase Velasco (2003).

13 Pérez Caltendey (2003) resalta la importancia que tienen los incentivos de alcance sectorial en las economías caribeñas, particularmente en los países miembros de la Organización de Estados del Caribe Oriental, así como en Barbados y sobre todo en Guyana, que exhibe el conjunto de incentivos más amplio de la región. Esos incentivos se orientan fundamentalmente hacia los sectores manufactureros y de servicios, en particular hoteles y turismo (Hendrickson, 2003).

${ }^{14}$ Ese enfoque fue desarrollado a partir de Porter (1990) y se concretó en los trabajos de la Monitor Company en los países andinos a comienzos del decenio de 1990 y en el proyecto "Centroamérica en el siglo XXI: Una agenda para la competitividad y el desarrollo sostenible", coordinado por INCAE/Centro Latinoamericano para la Competitividad y el Desarrollo Sostenible (CLADS) a mediados de ese decenio. 
que se ha utilizado en el presente artículo. En su aplicación, esos enfoques se traducían en la negociación y puesta en marcha de acuerdos sectoriales, generalmente a lo largo de cadenas de valor, entre actores privados y el gobierno, donde este cumplía la función de catalizador o facilitador.

Finalmente, en Argentina, Chile y Uruguay no se trabajó sobre la base de políticas industriales ni de estrategias nacionales de competitividad. Se prefirieron las políticas llamadas horizontales, ${ }^{15}$ que buscaban no discriminar entre sectores y que se debían poner en práctica a través de incentivos a la demanda de las empresas, en contraste con los subsidios a la oferta que caracterizaban al modelo anterior. Cuando se presentaran problemas con clara dimensión sectorial, las políticas horizontales se concretarían a través de la concentración de sus instrumentos en la solución de tales problemas, sin que se considerara que por ello perdían su carácter básicamente neutral. En Chile fue donde se conceptualizó y puso en marcha con más fuerza este tipo de intervención, aunque en el país se mantuvieron por un largo período subsidios directos a los sectores forestal y minero, así como a las actividades exportadoras (Moguillansky, 2000).

A comienzos del decenio del 2000, a diferencia de lo que sucede en otras áreas de la política de desarrollo, no hay convergencia en la posición de los países de América Latina y el Caribe en materia de políticas sectoriales. Mientras en algunos el discurso rechaza con vigor esas políticas, aunque hay prácticas ad hoc de apoyo sectorial, en otros se las reconoce como válidas para aumentar la competitividad de actividades con potencial para penetrar en mercados externos o que enfrentan una fuerte competencia de las importaciones. Por lo demás, hay un doble estándar frente a esas políticas: países que niegan su utilidad, en especial cuando apoyan al sector manufacturero, las utilizan abiertamente, y sin intentos por legitimarlas, en numerosos rubros agrícolas y de servicios (como el turismo, por ejemplo).

Teniendo como base el análisis histórico que aquí se ha hecho sobre la formulación de políticas, se pue-

\footnotetext{
15 La expresión "políticas neutrales u horizontales", de uso generalizado en la región, oculta el hecho de que toda política termina favoreciendo más a unos sectores que a otros. Esto sucede porque esas políticas intentan hacer más eficiente el funcionamiento de los mercados de factores productivos que son utilizados en diferente proporción por los distintos sectores o productos. En algunos casos, políticas que se presentan como neutrales para que tengan más legitimidad, están desde un comienzo orientadas a sectores específicos. Esto suele suceder con las políticas de desarrollo tecnológico.
}

de clasificar u ordenar a los países de la región conforme a tres variables: el objeto de la intervención, la frecuencia o intensidad de ella, y la coordinación existente entre las medidas tomadas para llevar a cabo las políticas en el marco de una estrategia de alcance más amplio.

Según el objeto de la intervención, cabe distinguir — como se señaló anteriormente- tres tipos de países: i) los que han mantenido o incluso reactivado políticas sectoriales, ii) aquellos en los que el tema sectorial tiene fundamentalmente una dimensión de conglomerado productivo, y iii) los que no han adoptado ninguna de estas dos perspectivas y solo aceptan el uso de políticas consideradas horizontales, las que, sin embargo, se concentran algunas veces en un sector específico.

En estos tres tipos de países las políticas horizontales son ampliamente aceptadas. Lo que distingue a los primeros dos tipos son las políticas que aplican además de las horizontales. El cuadro 1 pone de relieve la existencia de préstamos de bancos de desarrollo e incentivos fiscales orientados a sectores específicos. ${ }^{16}$ En siete países de la región (Argentina, Brasil, Colombia, Costa Rica, El Salvador, Honduras y México) la banca pública de desarrollo provee créditos con enfoque sectorial, mientras que en 18 países se han establecido incentivos fiscales para beneficiar a sectores específicos. Solo en Colombia, Guatemala, Haití, Honduras, Paraguay y Suriname no existen tales incentivos. Una forma de incentivo aun más generalizada es la presencia de normas especiales que favorecen la instalación de zonas de libre comercio para la exportación o de industrias maquiladoras.

Del análisis del conjunto de esa información surge una primera diferencia con las prácticas vinculadas al modelo anterior. Mientras que entonces se privilegiaba la industria manufacturera, ahora este es uno de los sectores con menos peso. Las actividades más favorecidas han sido el turismo, sectores primarios como el del petróleo, el minero y el forestal, y servicios varios (que van desde infraestructura hasta cinematografía). La importancia de las políticas orientadas al sector agrícola varía apreciablemente entre los países de la región si se la mide por los gastos públicos destinados a implementarlas (incluyendo programas de fomento productivo, inversiones en infraestructura rural

\footnotetext{
${ }^{16}$ La información disponible no permite determinar el monto de los subsidios implícitos en las operaciones de crédito y en los incentivos fiscales.
} 
CUADRO 1

América Latina y el Caribe: Incentivos financieros

y fiscales a sectores específicos

\begin{tabular}{|c|c|c|}
\hline País & $\begin{array}{c}\text { Préstamos a sectores específicos, excepto } \\
\text { agricultura }\end{array}$ & $\begin{array}{l}\text { Incentivos fiscales a sectores } \\
\text { específicos }\end{array}$ \\
\hline Argentina & Bienes de capital & Minería, actividad forestal \\
\hline Bahamas & & $\begin{array}{l}\text { Hoteles, servicios financieros, cerveza y bebidas } \\
\text { alcohólicas }\end{array}$ \\
\hline Barbados & & $\begin{array}{l}\text { Servicios financieros, seguros, tecnologías de } \\
\text { información, turismo }\end{array}$ \\
\hline Belice & & Minería \\
\hline Bolivia & & Minería \\
\hline Brasil & $\begin{array}{l}\text { Petróleo, gas natural, textiles, prendas de vestir, } \\
\text { calzado, industria naval, electricidad, } \\
\text { telecomunicaciones, software, cinematografía }\end{array}$ & Ind. automotriz, electrónica \\
\hline Chile & & Actividad forestal, petróleo, materiales nucleares \\
\hline Colombia & Cinematografía & \\
\hline Costa Rica & Diversos sectores & Actividad forestal, turismo \\
\hline Ecuador & & Minería, turismo \\
\hline El Salvador & $\begin{array}{l}\text { Minería, servicios (turismo, transporte, software y } \\
\text { otros) }\end{array}$ & \\
\hline \multicolumn{3}{|l|}{ Guatemala } \\
\hline Guyana & & $\begin{array}{l}\text { Agroindustria, actividad forestal, minería, turismo, } \\
\text { pesca, construcción, tecnologías de la información } \\
\text { y de las comunicaciones (TIC) }\end{array}$ \\
\hline \multicolumn{3}{|l|}{ Haití } \\
\hline Honduras & Transporte, camarón & \\
\hline Jamaica & & $\begin{array}{l}\text { Cinematografía, turismo, bauxita, aluminio, } \\
\text { construcción de fábricas }\end{array}$ \\
\hline Nicaragua & & Turismo \\
\hline Panamá & & Turismo, actividad forestal \\
\hline \multicolumn{3}{|l|}{ Paraguay } \\
\hline Perú & & Turismo, minería, petróleo \\
\hline República Dominicana & & Turismo, agroindustria \\
\hline \multicolumn{3}{|l|}{ Suriname } \\
\hline Trinidad y Tabago & & Hidrocarburos, turismo, construcción \\
\hline Uruguay & & $\begin{array}{l}\text { Carne ovina, viñedos y vino, hidrocarburos, } \\
\text { imprenta, actividad forestal, industrial militar, } \\
\text { aerolíneas, diarios, estaciones de radio, teatros, } \\
\text { cinematografía }\end{array}$ \\
\hline $\begin{array}{l}\text { Venezuela } \\
\text { (República } \\
\text { Bolivariana de) }\end{array}$ & & $\begin{array}{l}\text { Hidrocarburos y compras de bienes de capital y } \\
\text { servicios para inversiones en sectores primarios } \\
\text { (petróleo, minería, agricultura y pesca). }\end{array}$ \\
\hline
\end{tabular}

Fuente: Elaboración propia sobre la base de Melo (2001, cuadro 3). 
y gastos sociales en áreas rurales). ${ }^{17}$ Por su parte, la banca pública de desarrollo hace una contribución importante al financiamiento de ese sector en países como Argentina, Brasil, Costa Rica, México o la República Dominicana (Acevedo, 2002). En general, el crédito se da en condiciones cercanas a las de mercado y se mantienen subsidios a las tasas de interés en programas para fortalecer la pequeña agricultura.

La proposición anterior debe ser matizada al considerar las carteras de crédito de los seis bancos de desarrollo de cinco países de la región. La actividad industrial continúa siendo la receptora de alrededor de la mitad de los créditos del Banco Nacional de Desarrollo Económico y Social (BNDES) de Brasil, del Banco Nacional de Comercio Exterior (BANCOMEXT) de México y del Banco de Comercio Exterior de Colombia (BANCOLDEX), mientras que su participación es de cerca del $25 \%$ del total de la cartera de la Corporación Financiera de Desarrollo (COFIDE) de Perú e inferior a $15 \%$ en las carteras del Banco Nacional de Costa Rica (BNCR) y de la Nacional Financiera (NAFIN) de México. Dado que los créditos del BANCOLDEX y el BANCOMEXT se orientan a financiar el comercio exterior, de las seis instituciones solo el BNDES tendría un papel destacado en el financiamiento de la actividad productiva del sector industrial orientado al mercado interno, cuyas operaciones superaron los 5.800 millones de dólares en el 2002 (CEPAL, 2004a, cuadro 8.2). ${ }^{18}$

La segunda variable permite distinguir entre los países de la región según la frecuencia o intensidad con que llevan adelante políticas sectoriales: i) países que desarrollan una amplia gama de políticas a nivel sectorial (como Brasil, Colombia, Guyana, México, Uruguay, la República Bolivariana de Venezuela); ii) países que las desarrollan solo para unas pocas actividades (Bolivia, Chile, Perú, la República Dominicana, entre otros), y iii) países en los cuales esas políticas son casi inexistentes (como Haití, Paraguay, Suriname). Más allá de la información que proporciona el cuadro 1, la intensidad con que se llevan a cabo las políticas sectoriales también se puede detectar a partir de acciones que no implican subsidios fiscales y financieros, como en el caso de Colombia, donde hay una muy activa

\footnotetext{
${ }^{17}$ En Chile y México, los gastos anuales por productor alcanzaron los 900 dólares en el año 2000, mientras que en Bolivia fueron inferiores a 50 dólares. En ese mismo año, el gasto agrícola como proporción del PIB sectorial fue de $35 \%$ en México, $21 \%$ en Chile y un poco más de $5 \%$ en Bolivia (Kerrigan, 2001).

${ }_{18}$ Es probable que el rubro "Otros" de la cartera de NAFIN, que representa el $97 \%$ de ella, incluya operaciones orientadas al sector productivo interno.
}

política de acuerdos sectoriales que no contemplan estos tipos de incentivos, o de El Salvador, país que tiene una activa política de apoyo a los conglomerados productivos (Alonso, 2003).

En México, a partir de la instauración de la Política Económica para la Competitividad en el 2002, se han definido 12 ramas productivas prioritarias que serían objeto de programas sectoriales: cuatro de ellos están en operación (para la cadena de fibras, textiles y prendas de vestir; para cuero y calzado; para la industria electrónica y de alta tecnología, y para software) y hay avances en algunos programas para la industria automotriz, la maquiladora de exportación y la química. ${ }^{19}$ Por su parte, en noviembre de 2003 el gobierno de Brasil dio a conocer las Directrices de Política Industrial, Tecnológica y de Comercio Exterior, en las que expone sus opciones estratégicas sectoriales en cuatro actividades productivas que hacen uso intensivo de conocimiento: las de semiconductores, software, fármacos y medicamentos, y bienes de capital, y creó una entidad destinada a coordinar la puesta en marcha de esa política, la Agencia brasileña de desarrollo industrial. ${ }^{20}$

En algunos países - Costa Rica, Perú y Uruguay, entre otros - las medidas de fomento se han aplicado de manera aun más focalizada y se ha llegado incluso a apoyar proyectos individuales de ciertas empresas. Algunos ejemplos son los incentivos a la inversión en megaproyectos de la minería peruana, ${ }^{21}$ las acciones del gobierno de Costa Rica para que INTEL se instalara en el país, ${ }^{22} \mathrm{o}$ las exoneraciones fiscales destinadas a

\footnotetext{
${ }^{19}$ Las cinco ramas restantes son aeronáutica, agricultura, turismo, comercio y construcción (Secretaría de Economía, 2003).

${ }^{20} \mathrm{El}$ documento respectivo indica que se seleccionaron esos sectores porque i) presentan dinamismo creciente y sostenido; ii) responden por cuotas importantes de las inversiones internacionales en investigación y desarrollo; iii) abren nuevas oportunidades de negocios; iv) se relacionan directamente con innovación de procesos, productos y formas de uso; v) aumentan la densidad del tejido productivo, y vi) son importantes para el futuro del país y tienen potencial para el desarrollo de ventajas comparativas dinámicas (Ministério do Desenvolvimento, Indústria e Comércio Exterior, 2003, p. 16).

${ }^{21}$ En el caso de Perú, las principales políticas de apoyo al sector minero en el decenio de 1990 fueron: promoción y garantías a la inversión extranjera directa; privatización de empresas estatales; aprobación de una ley marco que garantizaba la libre iniciativa y las inversiones privadas; estabilidad tributaria, cambiaria y administrativa; modernización del proceso de concesiones mineras; beneficios fiscales (el impuesto a la renta no grava a utilidades reinvertidas); incentivos fiscales a la inversión en megaproyectos (exenciones del impuesto sobre los ingresos y recuperación anticipada del impuesto general sobre las ventas). Véase Fairlie (2003).

22 Véase Alonso (2003).
} 
respaldar proyectos declarados de interés nacional en Uruguay. ${ }^{23}$

La tercera variable - el nivel de coordinación entre las medidas aplicadas - obliga a considerar una dimensión adicional, inherente a la lógica de las políticas en que se basan: su integración o no integración en una estrategia nacional más amplia. Cabe distinguir entonces tres tipos de países: aquellos con acciones frecuentes en el marco de estrategias de intervención pública explícitas, generalmente expresadas en planes o programas oficiales (Brasil, Colombia, El Salvador y México, por ejemplo); países con intervenciones frecuentes, pero sin una estrategia explícita (Costa Rica, Uruguay) y países con intervenciones esporádicas (la gran mayoría).

Las tres variables consideradas han sido muy estables en cada país a lo largo del tiempo, lo que indica capacidad y experiencia en la formulación y aplicación de políticas. Los cambios de gobierno, incluso cuando significaron una fuerte ruptura con el pasado político del país, como en México en el año 2000 o Uru- guay en el 2005, no han modificado grandemente las posturas respecto a las políticas. Dos ejemplos, aunque en direcciones opuestas, son el poco peso que continúan teniendo las políticas sectoriales en Chile, y la continuidad de los esfuerzos por formular y mantener acuerdos sectoriales (convenios de competitividad exportadora) en Colombia a lo largo de las administraciones de los presidentes Samper, Pastrana y Uribe, acuerdos que involucran a 41 cadenas productivas y sectores responsables del $86 \%$ de las exportaciones no tradicionales. $^{24}$

La significación de esta muestra de madurez institucional, con todo lo positiva que es, no debe ser exagerada, pues la experiencia regional también abunda en ejemplos de programas establecidos para paliar problemas de eficiencia que han hecho crisis. ${ }^{25}$ Incluso la política para la industria automotriz brasileña tiene elementos de rescate de un sector en crisis que no puede soportar la competencia externa o, como se llamaba en el decenio de 1980, de reconversión industrial (Bonelli y Motta Veiga, 2003).

\section{III}

\section{Líneas de acción e instrumentos}

Como se ha reiterado en la literatura especializada, ${ }^{26}$ las políticas de competitividad en la región, incluso las de alcance fundamentalmente sectorial, se han preocupado mucho más de incrementar la eficiencia de sectores existentes que de crear sectores, lo que es consistente con la búsqueda de una mayor penetración en los mercados internacionales, principalmente sobre la

\footnotetext{
${ }^{23}$ La ley de inversiones de 1998 promueve inversiones específicas mediante la declaración del Poder Ejecutivo de que un proyecto es de interés nacional. Los beneficios pueden ser generales o favorecer solo al proyecto en cuestión (por ejemplo, exoneración del impuesto al patrimonio sobre bienes inmuebles). Los beneficios generales pueden ser automáticos (por ejemplo, exoneración del impuesto al patrimonio sobre bienes muebles destinados al ciclo productivo) o facultativos (que aún no se reglamentaban a mediados del 2003). Véase Scarone (2003).

24 De esas cadenas y sectores, 31 son nacionales y 10 regionales; 29 corresponden a bienes y 12 a servicios. No todos son programas para cadenas productivas en sentido estricto, pues están dirigidos a sectores como los de la papa, camarón de cultivo, atún, camarón de pesca, flores, café y banano. El concepto de cadena productiva no se aplicó con rigor porque la firma de dichos acuerdos obedeció a "razones prácticas" que buscaban la movilización de los empresarios (Velasco, 2003).
}

base de ventajas comparativas estáticas (mano de obra no calificada y recursos naturales). Esto se ha dado tanto en países con una estructura productiva diversificada (Brasil y México, entre otros), como en países con estructuras más especializadas. De los primeros se podría decir que son pocos los sectores inexistentes y que las políticas sectoriales se detectan a nivel de productos específicos. Si bien eso es cierto, la evidencia apunta a que, sobre todo en Brasil y en menor grado en México, ${ }^{27}$ las medidas de tipo sectorial se han concentrado en fortalecer y expandir sectores ya instalados, siendo el caso más notorio el de la industria automotriz, que se indicó anteriormente.

La creación de actividades nuevas aparece esporádicamente como objetivo de política; con ese propósito

\footnotetext{
25 Véase Scarone (2003) respecto al caso de gran parte de las políticas en Uruguay, y Villagómez (2003) respecto al programa para la industria electrónica 2002-2010 en México.

${ }^{26}$ Véase BID (2001), Melo (2001) y Peres (1997).

${ }^{27}$ Respecto a Brasil, véase Bonelli y Motta Veiga (2003); respecto a México, véase Unger (2003) y Villagómez (2003).
} 
se han seguido dos principales líneas de acción: las negociaciones comerciales internacionales para asegurar el acceso a los mercados, sobre todo mediante tratados bilaterales o multilaterales de libre comercio, y la atracción de inversión extranjera directa (IED) para desarrollar plataformas de exportación, incluyendo las actividades de zona franca y las maquiladoras.

La atracción de inversión extranjera directa ha sido el principal mecanismo por el cual se han creado nuevos sectores en la mayoría de los países de la región. Las medidas en ese sentido van desde la profundización de la plataforma exportadora de México en el marco del Tratado de Libre Comercio de América del Norte (automóviles y sus partes, electrónica y vestimenta), pasan por las actividades más elementales de maquiladoras de primera generación en algunos países de Centroamérica y el Caribe (prendas de vestir), y llegan hasta las inversiones en privatización de servicios y sectores primarios en países de América del Sur (Mortimore, 2000; Peres y Reinhardt, 2000). Las diferentes combinaciones de estrategias de las empresas transnacionales inversoras, por un lado, y de políticas públicas de alcance sectorial, por otro, han determinado en gran medida las actividades que han conducido a la diversificación de las estructuras productivas, aunque con las limitaciones derivadas del bajo valor agregado que resulta de la concentración en actividades de ensamblaje y de la poca articulación con el resto de la economía nacional respectiva.

Los instrumentos que se han utilizado para atraer inversión extranjera se pueden clasificar en tres grandes grupos (Mortimore y Peres, 1998): i) incentivos, principalmente del tipo zona franca y fiscales, ii) normas que generen un entorno eficiente -Estado de derecho, transparencia, acceso asegurado a mercados internacionales, buena infraestructura, etc. - y iii) creación de factores de producción especializados, en particular mano de obra calificada. Los países de la región han aplicado con diferente intensidad estos tres tipos de instrumentos; sin embargo, los que suelen predominar, con pocas excepciones, son los dos primeros.

Además de los instrumentos específicos para atraer inversión extranjera, se han utilizado otros dos que se dirigen a cualquier tipo de inversión (nacional o extranjera). Ellos son los incentivos financieros y fiscales indicados en el cuadro $1 \mathrm{y}$ un amplio grupo de medidas con las cuales los gobiernos buscan establecer entornos competitivos para el funcionamiento de las empresas (defensa de la competencia y regulación de sectores monopólicos), disminuir los costos de tran- sacción (reducción de controles administrativos, entre otros) o permitir el aprovechamiento de economías de escala mediante la acción colectiva de las empresas (acuerdos sectoriales a lo largo de cadenas productivas, apoyo a la asociación entre empresas, etc.).

Según el grado de aceptación que han logrado, las políticas formuladas en la región se pueden agrupar así: políticas ganadoras, políticas perdedoras y políticas emergentes (Peres, 1997). Las políticas ganadoras son las que han sido generalmente aceptadas por los gobiernos, es decir, que tienen gran legitimidad. Además de las políticas para fomentar las exportaciones y para atraer inversión extranjera directa, que ya se han mencionado, este grupo incluye las políticas para promover el desarrollo tecnológico, la formación de recursos humanos, las empresas pequeñas y microempresas - generalmente bajo la forma de medidas de apoyo a la constitución o consolidación de redes o conglomerados empresariales - y el desarrollo productivo a nivel local o subnacional, estando las dos últimas estrechamente vinculadas. La aceptación de estas políticas se debe a su presunta neutralidad por el hecho de incidir sobre mercados de factores productivos (tecnología y formación) o a su también presunto impacto positivo sobre la generación de empleo, especialmente a nivel subnacional o local.

Las políticas perdedoras, por su parte, son las que están en contradicción más clara con el modelo de desarrollo vigente, en particular con la apertura comercial y la reducción del déficit del sector público. En ellas se incluyen los subsidios fiscales directos, el crédito dirigido y con tasas de interés subsidiadas, los aranceles sobre comercio exterior y el uso del poder de compra del Estado. Sobre este último, la situación varía de un país a otro. Mientras en algunos se utiliza ese instrumento a nivel nacional o subnacional, como en el mencionado programa de apoyo a la producción de software en México, en otros está fuera de la gama de políticas aplicables, pues se considera que iría en contra de los objetivos de eficiencia y transparencia del gasto.

Por último, las políticas emergentes —entre otras, la defensa de la competencia, la mejora de los regímenes de gobernanza empresarial, la regulación de sectores de infraestructura cuyos mercados no operan con eficiencia, o la responsabilidad social de las empresas- están adquiriendo creciente legitimidad, pero aún se encuentran en proceso de maduración y, dentro de la región, su desarrollo varía mucho entre países. En algunos de ellos hay legislación moderna e instituciones relativamente sólidas que implementan tales políticas, 
mientras que en otros aún se hallan en instancias de debate y decisión o no son parte significativa de la agenda.

Más allá de las diferencias nacionales, se comprueba en la región una fuerte convergencia del contenido de los documentos de política en la última década. Esa convergencia se da alrededor de cuatro elementos básicos: i) el énfasis en el aumento de la competitividad en el ámbito internacional, ii) la generalización de la legitimidad de instrumentos de tipo hori- zontal o neutral, que, como ya se señaló, distan de serlo ex post, iii) el apoyo a las empresas pequeñas y microempresas, fundamentalmente por razones vinculadas a su capacidad de generar empleo y iv) la focalización en áreas económicas subnacionales o locales. Los programas de apoyo a conglomerados productivos son la concreción más notoria de al menos tres de estos elementos, siendo posiblemente el programa brasileño de apoyo a los arreglos productivos locales (APL) su expresión más importante en América Latina y el Caribe.

\section{IV}

\section{Evaluación de la implementación e impacto de las políticas industriales}

Las evaluaciones de la implementación y los efectos de las políticas industriales están limitados no solo por la información disponible, sino por el hecho de que, hasta hace muy poco, dichas políticas rara vez señalaban expresamente criterios y mecanismos para su evaluación. A esto se agrega la complejidad técnica de evaluar políticas que tienen múltiples objetivos y líneas de acción.

\section{Evaluación de la puesta en práctica de las políticas}

Si bien hay datos sobre los recursos financieros que se destinaron a algunas políticas, que en realidad constituían programas o proyectos, ${ }^{28}$ la información es insuficiente para evaluar la implementación en su conjunto. Pese a ello se constata que, con algunas excepciones, el grado de aplicación de las políticas en América Latina y el Caribe continúa siendo bajo, como ya había indicado Peres (1997); particularmente claro en este sentido es el análisis de Alonso (2003) sobre la situación de los cinco países centroamericanos y el de Fairbanks y Lindsay (1997) sobre los países andinos que concibieron estrategias de competitividad en torno al concepto de conglomerados productivos.

\footnotetext{
${ }^{28}$ Los casos mejor documentados se refieren a los montos asignados a las políticas agrícolas, en particular en el marco de grandes programas como PROCAMPo, la Alianza para el Campo y el Programa de Apoyo a la Comercialización en México. Véase Kjöllerström (2004), Villagómez (2003) para México y Scarone (2003), para Uruguay.
}

Según dichos estudios, las causas de las fallas generalizadas en la implementación de las políticas —es decir, de las "fallas del gobierno" - y la consiguiente brecha entre lo que se decide y lo que se ejecuta son de diversas clases, como se muestra a continuación.

\section{a) Objetivos no operacionales o inalcanzables}

La inclusión de objetivos no operacionales o inalcanzables en la formulación de las políticas transfiere la decisión real de ponerlas en marcha a la etapa de asignación de recursos presupuestarios. En estos casos, el problema reside en que, por fallas en su formulación, las políticas suelen tener más de declaración que de instrumento para asignar recursos. En este sentido, la evaluación de los factores de éxito en los 41 acuerdos sectoriales colombianos muestra que: i) los convenios con compromisos bien estructurados, cuantificables y por plazos definidos son más fáciles de seguir y cumplir; ii) los convenios con compromisos poco numerosos y sencillos obtienen mayores logros; iii) el liderazgo y el poder de decisión de las personas detrás de los convenios son fundamentales, y iv) las cadenas productivas que habían sido apoyadas desde antes de los convenios lograron mejores resultados. ${ }^{29}$ La práctica en la región suele no tener en cuenta estos factores de éxito. Así, los documentos de política tienden a desembocar en verdaderas listas de necesidades y objetivos. Si bien la multiplicidad de

\footnotetext{
${ }^{29}$ Véase Velasco (2003).
} 
objetivos puede deberse a la acción de muchos agentes en sociedades complejas, también refleja cierta incapacidad de establecer prioridades y de construir consenso alrededor de unas pocas, pero que puedan llevarse a la práctica.

\section{b) Escasez de recursos humanos y financieros}

La escasez de recursos humanos y financieros para poner en marcha las políticas, que es especialmente grave en los países más pequeños y más pobres, hace que muchas veces se dependa de recursos externos (préstamos o ayuda) para formular las políticas y más aun para aplicarlas. Además, al enunciar las políticas no se suele considerar su costo y el correspondiente financiamiento, ateniéndose una vez más a que "primero decidimos y después vemos qué hacemos y con qué recursos".

\section{c) Poca capacidad institucional}

Casi todos los países de la región tienen poca capacidad institucional para implementar políticas, incluidas algunas bastante sencillas. La dificultad es mayor cuando se trata de poner en marcha políticas que reflejan más la "mejor práctica internacional" que las necesidades de los países interesados en aplicarlas. Esto se traduce en formulaciones de política desapegadas de la realidad que, peor aun, muchas veces son impulsadas por instancias estatales con poca gravitación en la estructura de poder de los gobiernos o por asociaciones empresariales con baja representatividad y poco peso económico y político. El problema se agudiza porque en la región normalmente están separadas las instancias de formulación y las de implementación de las políticas. Si bien los países pueden acrecentar su capacidad institucional a lo largo del tiempo - y en la región algunos lo han hecho-, la creación e innovación institucionales exigen estabilidad de objetivos por períodos más extensos que los cuatro a seis años habituales de los gobiernos en América Latina y el Caribe, así como recursos financieros que confieran capacidad de acción. En este sentido, la gran disparidad de cargas fiscales entre los diversos países de la región — desde menos de $10 \%$ a más de $30 \%$ del PIB — introduce diferencias estructurales en sus posibles logros en este terreno.

\section{d) Precariedad de los acuerdos público-privados}

Los acuerdos entre el gobierno y el sector privado para poner en marcha las políticas son precarios, lo que se comprueba en el momento de materializar gastos del sector público o compromisos de inversión y gastos de contrapartida del sector privado. Más aun, proliferan los planes y programas que solo se elaboran para responder a presiones políticas de actores económicos, para solicitar financiamiento internacional o para cumplir con disposiciones legales o constitucionales. El sector empresarial, que defendió con mucha fuerza las políticas de protección hasta fines del decenio de 1970, no muestra igual fortaleza para llevar adelante políticas de diversificación y de mejoramiento de la especialización productiva en los países de la región. ${ }^{30}$

\section{e) Debilidad de las señales económicas}

Los problemas de implementación se conjugan, en el caso de las políticas industriales, con la debilidad de las señales económicas que emiten los programas destinados a expandir o crear actividades. Frente al vigor y claridad de la señal asociada a la protección comercial, que permitía fijar precios internos y maximizar la rentabilidad y que era típica del modelo de industrialización mediante la sustitución de importaciones, actualmente se ofrece al empresario, en el mejor de los casos, un conjunto de señales difíciles de interpretar y de traducir en medidas concretas y cuyo efecto sobre la rentabilidad es incierto. No debe sorprender entonces que la percepción de que "las políticas no funcionan" sea tan generalizada.

Las fallas de implementación y la percepción de que "las políticas no funcionan" afectan la legitimidad de las políticas industriales y el interés que puedan tener en ellas sus principales destinatarios, los empresarios. Esto lleva a una situación paradójica: los empresarios consideran que los recursos disponibles para poner en vigor las políticas son escasos $\mathrm{y}$, sin embargo, no los utilizan en su totalidad. Encontrar la manera de superar las fallas de implementación y hacer que las políticas funcionen es uno de los principales desafíos que encaran las estrategias de desarrollo.

Pese a las fallas de implementación mencionadas, ha habido avances en las relaciones entre las autoridades públicas y las asociaciones (o cámaras) empresariales en la etapa de formulación de políticas y, en algunos pocos casos, en la de su aplicación. El diálogo público-privado se ha venido fortaleciendo desde los comienzos del decenio de 1990, aunque subsisten algunas situaciones de conflicto, y ha avanzado hasta

\footnotetext{
${ }^{30}$ Por otra parte, las discrepancias entre el gobierno y el sector privado, si bien han menguado, distan de haber desaparecido, como señala Alonso (2003) respecto de Guatemala y Scarone (2003) respecto de Uruguay.
} 
el punto de que a menudo el liderazgo en las propuestas de política ha provenido de entidades empresariales. Así ha sucedido en el caso de la Asociación Nacional de Industriales (ANDI) en Colombia, la Cámara Nacional de la Industria de Transformación (CANACINTRA) en México, la Asociación de Industriales de República Dominicana (AIRD), la Cámara de Industrias de Costa Rica (CICR) o la Federación de Cámaras Industriales de Centroamérica (FECAICA), entidad que en el decenio de 1990 impulsó una agenda de modernización industrial en esa región. En estos y otros países es posible entonces hablar de una corresponsabilidad público-privada en la formulación de políticas, más que de una simple concertación de políticas (Peres, 1997).

Las asociaciones empresariales también han participado con diferente intensidad en foros de negociación para establecer medidas de apoyo a la competitividad, tales como el Consejo Nacional de Competitividad en Colombia, el Foro de Desarrollo Productivo en Chile o las "cámaras sectoriales" en Brasil. ${ }^{31}$ En algunos casos, han llegado incluso a hacer propuestas de largo plazo tendientes a dar estabilidad a las políticas más allá de los períodos de gobierno; así sucedió, por ejemplo, con la Visión 2020 impulsada por la Confederación de Cámaras Industriales de los Estados Unidos Mexicanos (CONCAMin).

Mucho más débil ha sido la participación de otras entidades de la sociedad civil en la formulación de las políticas. Si bien los sindicatos de trabajadores han estado en los foros de concertación, su presencia no ha sido determinante de la dinámica de esos foros, con pocas salvedades; una de las excepciones fue el papel desempeñado por la organización sindical en la "cámara sectorial" de la industria automotriz en Brasil. Otras entidades han tenido un papel aun menor, siendo una excepción la participación del sector académico en el Consejo Nacional de Competitividad en Colombia.

\section{Evaluación del impacto de las políticas}

Lo que se ha hecho para evaluar los efectos de las políticas industriales es tanto o más limitado e insatisfactorio que lo hecho para evaluar la implementación de esas políticas. Hay evaluaciones de algunos programas específicos, como los de apoyo a las pequeñas

\footnotetext{
31 En Brasil, la expresión "cámara sectorial" no indica una asociación empresarial, sino un espacio de negociación tripartito (Estado, empresarios y trabajadores).
}

empresas en Chile, ${ }^{32}$ así como evaluaciones generales sobre lo que pasó después de aplicar las políticas, sin aducir argumentos que indiquen que ellas hayan sido la causa de los hechos que se relatan. Algunas evaluaciones de este tipo se refieren al aumento de las exportaciones no tradicionales de Colombia provenientes de cadenas productivas con acuerdos sectoriales (Velasco, 2003), el incremento de las exportaciones mineras de Perú (Fairlie, 2003), las mejoras del ingreso de los productores rurales e incluso de la productividad de sus predios, a partir de los grandes programas agrícolas mexicanos (Villagómez, 2003) o la discusión sobre si hubo o no aumento de los ingresos autónomos de los productores apoyados por el Instituto Nacional de Desarrollo Agropecuario (INDAP) en Chile (Kjöllerström, 2004).

¿Qué se puede hacer para superar estos problemas de discrepancia entre lo que se decide y enuncia, y lo que se hace y evalúa? Conviene profundizar en tres líneas de acción, que no se excluyen entre sí y parecen promisorias.

En primer lugar, la formulación de políticas debe ir acompañada, no seguida, por consideraciones explícitas sobre las instituciones que las han de ejecutar. Esto implica que los interesados en la política industrial deberán incursionar en los temas de la reforma del Estado. La estructura de este sigue respondiendo a realidades organizadas en sectores y subsectores productivos, mientras se busca aplicar políticas de alcance sistémico o transversal que, por definición, abarcarán más de un sector o más de una entidad ejecutora. Esto tiene particular importancia en el caso de políticas que evolucionaron desde lo estrictamente sectorial a lo de alcance general, como las estrategias de apoyo a la difusión y el uso de las tecnologías de la información y las comunicaciones, cuyo carácter transversal ya se ha señalado. La reforma del Estado y el consiguiente desarrollo institucional no son temas cercanos a los especialistas en organización industrial, pero deben ser encarados para reducir las fallas de implementación.

Dada la escasez de recursos humanos calificados en las áreas del Estado vinculadas a la implementación de las políticas, una segunda línea de acción sería la de trasladar a esas áreas personal altamente calificado que esté trabajando en la formulación de las políticas. Esto no iría en detrimento de los esfuerzos por

\footnotetext{
${ }^{32}$ Para ese país, véase las evaluaciones a los programas de fomento presentadas en Silva y Sandoval (2003).
} 
construir capacidades, puesto que tales esfuerzos son necesariamente de largo plazo, mientras que la reasignación de recursos humanos puede ser de corto plazo. Obviamente, tal reasignación no dejará de tener costos elevados, tanto en términos de eficiencia como de carreras personales, pero es una alternativa que merece considerarse si se acepta el diagnóstico de que hay una gran diferencia entre lo que se dice y lo que se hace.

Una tercera línea de acción es la de desarrollar y fortalecer a los operadores de política, es decir, a instituciones y personas que combinen las capacidades de formulación y de acción. Con tal fin, se puede reforzar las instituciones públicas, buscar líderes en el sector privado y fortificar a agentes intermedios como las asociaciones empresariales y las organizaciones no gubernamentales.

El desarrollo institucional de largo plazo dentro del Estado es una realidad en los ministerios a cargo de la política macroeconómica y en los bancos centrales de América Latina y el Caribe. También en los sectores agrícolas y extractivos muchos países de la región han creado y mantenido instituciones vigorosas, como la Empresa Brasileira de Pesquisa Agropecuária (EMBRA-

\section{V}

\section{Conclusiones}

Este trabajo muestra que los países de América Latina y el Caribe tienen opciones de formular políticas para mejorar su especialización productiva y que las han utilizado. En la región se han concretado tres tipos de políticas, que han resultado de los objetivos, la experiencia y las capacidades económicas e institucionales de cada país. En particular, se han concebido medidas que han ido desde la puesta en marcha de políticas industriales en sentido estricto hasta la formulación de políticas básicamente horizontales concentradas en determinados sectores, pasando por el fomento de los conglomerados productivos con un enfoque de cadena de valor (Porter, 1990).

Las políticas industriales son el meollo de las estrategias de especialización o diversificación. Al plantear estrategias de esta índole hay cuatro aspectos importantes que considerar: los criterios para seleccionar los sectores que se promoverán, los instrumentos de política disponibles, las restricciones
PA) y los institutos del petróleo en México y la República Bolivariana de Venezuela. Esa experiencia puede y debe emularse en las áreas ligadas al desarrollo de otros sectores productivos. El liderazgo privado en materia de políticas ha sido eficiente en algunos casos (por ejemplo, en la formación de algunos conglomerados productivos a nivel local), y debe ser utilizado siempre que sea posible, pero en la región se ha visto que es difícil de sistematizar y que no se distribuye en forma que responda a las necesidades de implementación. Así, sectores económicamente débiles, que necesitan grandes esfuerzos de los operadores de política, tienden a tener liderazgos también débiles. El fortalecimiento de entidades intermedias de implementación ha sido una estrategia exitosa en países como Chile, donde se la ha utilizado para llevar a cabo los programas de fomento de la articulación productiva (PROFO), aunque no dejan de estar presentes los previsibles problemas de selección adversa y riesgo moral.

Ninguna de estas acciones es una panacea ni fácil de poner en práctica. Pero abren alternativas y merecen ser exploradas desde perspectivas que combinen las dimensiones económica, institucional y de gestión.

impuestas por el tamaño de los mercados nacionales y las capacidades acumuladas de los distintos países de la región, y la voluntad política de llevar a cabo este tipo de acciones.

La selección de sectores debe partir por reconocer que no hay criterios universales para decidir qué actividades han de ser promovidas. Sin embargo, existe una extensa experiencia internacional que muestra que, de hecho, los países han elegido y siguen eligiendo sectores y que lo hacen siguiendo unos pocos criterios más o menos precisos. Entre esos criterios destacan el contenido de conocimiento de las actividades en cuestión, su dinamismo en el mercado internacional debido a una elevada elasticidad con respecto al ingreso del mundo y en especial de los países desarrollados, y el potencial de crecimiento de su productividad. A estos criterios se agregan otros relacionados con el carácter estratégico de ciertas actividades, fundamentalmente por su peso en el producto, las exportaciones o el 
empleo, generalmente a nivel nacional, pero también a nivel local o subnacional. El examen de las políticas en las secciones anteriores ilustra ampliamente el uso de esos criterios, no siempre explícito, en los países de la región.

A partir del decenio de 1980 se ha ido utilizando cada vez más la dimensión tecnológica para definir el alcance de las políticas industriales. Aunque tradicionalmente un grupo de actividades ha sido considerado un "sector" cuando todas ellas producen bienes o servicios con alta elasticidad-precio cruzada, es posible también definir como "sector" a actividades que comparten una trayectoria tecnológica (Robinson, 1953): así, se habla de sector aeroespacial, sector de biotecnología o sector de tecnologías de la información y de las comunicaciones. Para el fomento de las actividades que comparten una tecnología determinada, hay tanto experiencias centradas en políticas horizontales como otras en las que se interviene directamente a nivel de empresas, segmentos de mercado o redes de conocimiento. Así como en materia de articulación de las actividades productivas las políticas industriales tendieron a concretarse en el fomento de los conglomerados productivos, en el campo de la tecnología prácticamente se confunden con las políticas de innovación o desarrollo tecnológico.

En la medida en que las políticas adquieren alcance sistémico, su impacto sobre las condiciones de competitividad del conjunto de la economía demanda especial atención. Los mayores costos asociados a las etapas iniciales de las curvas de aprendizaje no deben tener una magnitud que ponga en riesgo la competitividad de las empresas usuarias de los nuevos bienes o servicios, particularmente si esas empresas están fuertemente orientadas al comercio exterior. No es fácil alcanzar el equilibrio entre apoyar la diversificación del aparato productivo interno y aprovechar las oportunidades de importar bienes de capital e insumos más baratos o de mejor tecnología; ese equilibrio solo puede buscarse a partir de la experimentación y de métodos de tanteo, es decir, a través de políticas de base pragmática más que doctrinaria. Como las políticas pragmáticas suelen ser reactivas, un importante desafío que enfrenta la región es el de combinar el pragmatismo con políticas mucho más proactivas.

Los instrumentos disponibles para poner en práctica este tipo de políticas son conocidos y están presentes en la formulación de políticas en los países de América Latina y el Caribe. Pero ahora, a diferencia de lo que ocurría antes en la región y fuera de ella, se opera en el marco de economías abiertas y no es posi- ble usar instrumentos de protección comercial generalizada y permanente. Esta restricción debilita la señal económica (rentabilidad esperada) que se envía a los potenciales inversionistas en las nuevas actividades y hace que parte significativa del costo de las actividades de fomento deba recaer en el Estado. Esto conlleva problemas, tanto en lo que hace a la determinación de las prioridades para asignar recursos presupuestarios, como a la estabilidad de esos recursos en períodos de restricción fiscal. El sostenimiento de los mecanismos de fomento en el largo plazo, incluso más allá de un período de gobierno, sigue siendo un reto que los países de la región aún no han podido encarar con éxito. Otro poderoso instrumento de política sectorial, la inversión directa del Estado, está fuera de la agenda de gran parte de los países de la región; pero los grados de libertad en esta materia son grandes, como lo muestran diversos casos, particularmente a nivel local o subnacional. La experiencia de la región parece indicar que los conjuntos de políticas que se han aplicados hasta ahora, cuyos efectos acumulativos aún no han sido evaluados, no han tenido la fuerza inductora que en su momento tuvo la protección.

Se ha argumentado además que los países pequeños y con menores capacidades institucionales no deben ni pueden establecer políticas de alcance sectorial. Sin desconocer la importancia de utilizar el mercado interno para lograr economías de escala y aprendizaje, no se debe desconocer que en economías abiertas el tema pierde fuerza, como lo muestra la experiencia de numerosos países pequeños que funcionan como plataformas de exportación muy competitivas. Aunque la capacidad institucional sí es un requisito significativo, particularmente en el corto plazo, el hecho de que ella sea limitada no obliga a desechar las actividades de alcance sectorial, sino a concentrarlas en subsectores, segmentos o incluso productos que estén al alcance de las capacidades existentes. La alternativa es reducir la magnitud de los esfuerzos y no dar "saltos en el aire". En este sentido, la experiencia en la región con las políticas de fomento de los conglomerados productivos revela que incluso los países pequeños han podido formular políticas para mejorar su patrón de especialización.

Pese a estas consideraciones, en lo que se refiere a la voluntad política de llevarlas a cabo, las acciones sectoriales enfrentan una situación ambigua en la región. Su legitimidad es mayor en algunos países - aunque siempre inferior a la del anterior modelo de industrialización mediante sustitución de importaciones - y mucho menor o nula en otros. Sin embargo, 
incluso en países que no consideran legítimas las políticas sectoriales, se las practica de manera mucho más ad hoc y a menudo se aplican medidas específicas de apoyo a sectores en crisis. Dado que estas políticas son necesarias para avanzar en el desarrollo de la región, cabe preguntarse cuáles son las tareas necesarias para acrecentar su legitimidad.

Dos líneas de acción son prioritarias. Por un lado, es preciso mejorar la capacidad de implementación, reduciendo la brecha que existe entre la formulación de las políticas y la capacidad institucional para llevarlas a cabo; la persistencia de esa brecha daña la credibilidad de quienes establecen las políticas y, por ende, de las políticas mismas. Por otro lado, hay que avanzar considerablemente en la tarea de evaluar el impacto de las políticas en función de sus objetivos finales: crecimiento económico, progreso tecnológico, aumento de la productividad. Puesto que los recursos públicos son escasos, solo efectuando evaluaciones robustas será posible generar espacio para reasignar recursos desde otras áreas de política hacia éstas.

Estas reflexiones no son nuevas, pero sí son cruciales. ${ }^{33}$ Ha habido avances, como lo ilustra el Programa de Desarrollo Empresarial de México 20012006, que hace referencia explícita a metas cuantitativas, ${ }^{34}$ mostrando así un claro progreso respecto de programas anteriores. Sin embargo, los adelantos en el conjunto de la región son sin duda insuficientes. Esto afecta gravemente a políticas que deben competir por recursos fiscales con otras de gran legitimidad —como educación básica, salud pública o seguridad ciudadana- y que deben justificar su propia razón de ser. Por ser esenciales para diversificar el aparato productivo y posibilitar la aceleración del crecimiento de la productividad, las políticas industriales deben recuperar su legitimidad y para hacerlo tienen que mostrar sus efectos.

Desde una perspectiva más general, una cuestión crucial continúa abierta. Incluso si las políticas para diversificar la estructura productiva pudiesen demostrar técnicamente su capacidad de generar impactos positivos, no estaría claro cuáles serían los agentes sociales interesados en que esas políticas se generalizaran en los países de la región, es decir, los que respaldarían con sus recursos económicos y políticos iniciativas de esta índole, yendo más allá del fomento de los conglomerados productivos que, por lo demás y con pocas excepciones, distan de contar con recursos de significación. Las políticas industriales han tenido un lento retorno a América Latina y han podido operar, aunque en pequeña escala, en economías abiertas y con políticas macroeconómicas ortodoxas, pese a que ex ante se aceptaba que las políticas macroeconómicas ortodoxas serían incompatibles con la aplicación de políticas industriales. Para que estas políticas dejen de tener un peso solo marginal, los actores sociales, incluido el Estado, tendrán que hacerlas suyas y comprometer el respaldo de su poder y recursos. Y aquí surge la pregunta de fondo: ¿quién tiene o podría tener interés en políticas industriales proactivas y posee a la vez la fuerza y los recursos necesarios para modificar el patrón actual de especialización productiva?
Acevedo, R. (2002): ALIDE y el financiamiento de la agricultura y el medio rural, documento presentado en la Conferencia Rural "Desarrollo de las economías rurales en América Latina y el Caribe: manejo sostenible de los recursos naturales, acceso a tierras y finanzas rurales" (Fortaleza, Ceará, Brasil, 7 de marzo).

Alonso, E. (2003): Centroamérica: políticas para el fomento de los sectores productivos en Centroamérica, LC/L.1926-P, Santiago de Chile, Comisión Económica para América Latina y el Ca-

\footnotetext{
${ }^{33}$ Esta idea ya fue resaltada en Peres (1997), Stallings y Peres (2000) y Peres y Stumpo (2002).

${ }^{34}$ En el programa se plantea crear un sistema de evaluación pública que incluya indicadores estratégicos, mecanismos de control, mecanismos de coordinación y participación en la evaluación, rendición de cuentas periódica y un observatorio de medianas y pequeñas empresas y microempresas, como fuente de información (Secretaría de Economía, 2001, p. 56).
}

ribe (CEPAL). Publicación de las Naciones Unidas, $\mathrm{N}^{\circ}$ de venta: S.03.II.G.83.

Amsden, A. (1989): Asia's Next Giant, South Korea and Late Industrialization, Nueva York, Oxford University Press.

Balbi, S. (2003): Crise e divergência travam política industrial, Folha dinheiro, São Paulo, 2 de noviembre.

Banco Mundial (1993): The East Asian Miracle. Economic Growth and Public Policy, Washington, D.C.

BID (Banco Interamericano de Desarrollo) (2001): Competitividad: el motor del crecimiento, Progreso económico y social en América Latina. Informe 2001, Washington, D.C.

Bonelli, R. y P. Motta Veiga (2003): A dinâmica das políticas setoriais no Brasil na década de 1990: continuidade e mudança, proyecto regional CEPAL/GTZ "Una estrategia de desarrollo de clusters alrededor de recursos naturales: sus implicaciones sobre crecimiento, distribución y medio ambiente", Santiago de Chile, Comisión Económica para América Latina y el Caribe (CEPAL).

Castaldi, C. (2003): Essays on the Process of Economic Growth, Pisa, Italia, Scuola Superiore Sant'Anna. 
CEPAL (Comisión Económica para América Latina y el Caribe) (2003): Istmo centroamericano: los retos de la sustentabilidad en granos básicos, LC/MEX/L.554, México, D.F., Sede Subregional de la CEPAL en México.

(2004a): Desarrollo productivo en economías abiertas, LC/G.2234(SES.30/3), Santiago de Chile.

(2004b): La inversión extranjera en América Latina y el Caribe, 2003, LC/G.2226-P, Santiago de Chile. Publicación de las Naciones Unidas, $\mathrm{N}^{\circ}$ de venta: S.04.II.G.54.

(2005): Políticas públicas para el desarrollo de sociedades de información en América Latina y el Caribe, LC/W.19, Santiago de Chile, junio.

Cimoli, M., N. Correa y A. Primi (2003): Crecimiento y estructura productiva en economías abiertas: lecciones de la experiencia de América Latina, Santiago de Chile, Comisión Económica para América Latina y el Caribe (CEPAL).

Chang, H. (1994): The Political Economy of Industrial Policy, Londres, MacMillan Press.

Dussel Peters, E. (1999): La subcontratación como proceso de aprendizaje: el caso de la electrónica en Jalisco (México) en la década de los noventa, serie Desarrollo productivo, $\mathrm{N}^{\circ} 55$, LC/L.1183-P, Santiago de Chile, Comisión Económica para América Latina y el Caribe (CEPAL). Publicación de las Naciones Unidas, $\mathrm{N}^{\circ}$ de venta: S.99.II.G.16.

Fairbanks, M. y S. Lindsay (1997): Plowing the Sea: Nurturing the Hidden Resources of Growth in the Developing World, Boston, Massachusetts, Harvard Business School Publishing.

Fairlie, A. (2003): Políticas sectoriales y cadenas productivas en el Perú reciente, proyecto regional CEPAL/GTZ "Una estrategia de desarrollo de clusters alrededor de recursos naturales: sus implicaciones sobre crecimiento, distribución y medio ambiente", Santiago de Chile, Comisión Económica para América Latina y el Caribe (CEPAL).

FAO (Organización de las Naciones Unidas para la Agricultura y la Alimentación) (2001): Examen de las políticas sobre productos alimentarios básicos, Roma.

Hendrickson, M. (2003): Caribbean Tourism: Trends, Policies and Impact, 1985-2002, Santiago de Chile, Comisión Económica para América Latina y el Caribe (CEPAL).

Henry, L. (2003): Sectoral Policies: Information and Communications Technology in the Caribbean. Trends, Policies and Impact, 1985-2002, Santiago de Chile, Comisión Económica para América Latina y el Caribe (CEPAL), julio.

Kaldor, N. (1966): Causes of the Slow Rate of Growth of the United Kingdom, Cambridge, Cambridge University Press.

Kerrigan, G. (2001): Gasto público hacia el sector agrícola y desarrollo de las áreas rurales: ALC, tendencias y desafíos, Informe de consultoría, Santiago de Chile, Organización de las Naciones Unidas para la Agricultura y la Alimentación (FAO), agosto.

Kjöllerström, M. (2004): Competitividad del sector agrícola y pobreza rural: el papel de los gastos públicos en América Latina, serie Desarrollo productivo, No 155, LC/L.2137-P, Santiago de Chile, Comisión Económica para América Latina y el Caribe (CEPAL), mayo. Publicación de las Naciones Unidas, $\mathrm{N}^{\circ}$ de venta: S.04.II.G.66.

Krugman, P. (1994): The myth of Asia's miracle, Foreign Affairs, vol. $73, \mathrm{~N}^{\circ} 6$, Nueva York, Council on Foreign Relations, noviembre-diciembre.

Melo, A. (2001): Industrial Policy in Latin America and the Caribbean at the Turn of the Century, Working paper, $\mathrm{N}^{\circ} 459$, Washington, D.C., Banco Interamericano de Desarrollo (BID), agosto.

Ministério do Desenvolvimento, Indústria e Comércio Exterior (2003): Diretrizes de política industrial, tecnológica e de comércio exterior, Brasilia, 20 de noviembre.

Moguillansky, G. (2000): La inversión en Chile. ¿El final de un ciclo de expansión?, Santiago de Chile, Fondo de Cultura Económica.
Mortimore, M. (2000): Corporate strategies for FDI in the context of Latin America's new economic model, World Development, vol. $28, \mathrm{~N}^{\circ}$ 9, Amsterdam, Elsevier, septiembre.

Mortimore, M. y W. Peres (1998): Policy competition for foreign direct investment in the Caribbean Basin: Costa Rica, Jamaica and the Dominican Republic, serie Desarrollo productivo, N 49 (LC/G.1991), Santiago de Chile, Comisión Económica para América Latina y el Caribe (CEPAL).

Noland, M. y H. Pack (2002): Industrial Policies and Growth: Lessons from the International Experience, Documentos de trabajo, $\mathrm{N}^{\circ} 169$, Santiago de Chile, Banco Central de Chile, julio.

ONUDI (Organización de las Naciones Unidas para el Desarrollo Industrial) (2003): Informe sobre el desarrollo industrial correspondiente a 2002/2003: Competir mediante la innovación y el aprendizaje, ID/414, Viena. Publicación de las Naciones Unidas, $\mathrm{N}^{\circ}$ de venta: S.02.II.B.13.

Peres, W. (coord.) (1997): Políticas de competitividad industrial en América Latina y el Caribe en los años noventa, México, D.F., Siglo XXI Editores.

Peres, W. y N. Reinhardt (2000): Latin America's new economic model: micro responses and economic restructuring, World Development, vol. 28, $\mathrm{N}^{\circ}$ 9, Amsterdam, Elsevier, septiembre.

Peres, W. y G. Stumpo (2002): Las pequeñas y medianas empresas industriales en América Latina y el Caribe, México, D.F., Siglo XXI Editores.

Pérez Caltendey, E. (2003): Policies for Productive Development in Caribbean Economies, Santiago de Chile, Comisión Económica para América Latina y el Caribe (CEPAL), agosto.

Porter, M. (1990): La ventaja competitiva de las naciones, Buenos Aires, Javier Vergara Editor.

Robinson, J. (1953): Imperfect competition revisited, The Economic Journal, vol. 63, $\mathrm{N}^{\circ}$ 251, Oxford, Reino Unido, Blackwell Publishing.

Rodrik, D. (1995): Getting interventions right: how Korea and Taiwan grew rich, Economic Policy, N ${ }^{\circ}$ 20, Oxford, Reino Unido, Blackwell Publishing, abril.

Scarone, C. (2003): Las políticas sectoriales de Uruguay 1998-2002, proyecto regional CEPAL/GTZ "Una estrategia de desarrollo de clusters alrededor de recursos naturales: sus implicaciones sobre crecimiento, distribución y medio ambiente", Santiago de Chile, Comisión Económica para América Latina y e Caribe (CEPAL).

Secretaría de Economía (2001): Programa de Desarrollo Empresarial de México, 2001-2006, México, D.F. (2003): Política económica para la competitividad, México, D.F.

Sergeant, K., S. Racha y M. James (2003): The Petroleum Sector. The Case of Trinidad and Tobago. Trends, Policies and Impact. 1985-2002, proyecto regional CEPAL/GTZ "Una estrategia de desarrollo de clusters alrededor de recursos naturales: sus implicaciones sobre crecimiento, distribución y medio ambiente", Santiago de Chile, Comisión Económica para América Latina y el Caribe (CEPAL).

Silva, I. y C. Sandoval (2003): Políticas de desarrollo productivo en Chile, con especial referencia al nivel local y regional, Santiago de Chile, Comisión Económica para América Latina y el Caribe (CEPAL).

Stallings, B. y W. Peres (2000): Crecimiento, empleo y equidad. El impacto de las reformas económicas en América Latina y el Caribe, Santiago de Chile, Comisión Económica para América Latina y el Caribe (CEPAL)/Fondo de Cultura Económica.

Stigler, G. (1951): The division of labour is limited by the extent of the market, Journal of Political Economy, vol. 59, $\mathrm{N}^{\circ} 3$, Chicago, The University of Chicago Press.

Unger, K. (2003): Clusters industriales en México: especializaciones regionales y la política industrial, proyecto regional 
CEPAL/GTZ "Una estrategia de desarrollo de clusters alrededor de recursos naturales: sus implicaciones sobre crecimiento, distribución y medio ambiente", Santiago de Chile, Comisión Económica para América Latina y el Caribe (CEPAL).

Velasco, M.P. (2003): Una evaluación de las políticas de competitividad en Colombia, proyecto regional CEPAL/GTZ "Una estrategia de desarrollo de clusters alrededor de recursos naturales: sus implicaciones sobre crecimiento, distribución y medio ambiente", Santiago de Chile, Comisión Económica para América Latina y el Caribe (CEPAL).
Villagómez, A. (2003): Una revisión de la política sectorial en México: 1995-2003, proyecto regional CEPAL/GTZ "Una estrategia de desarrollo de clusters alrededor de recursos naturales: sus implicaciones sobre crecimiento, distribución y medio ambiente", Santiago de Chile, Comisión Económica para América Latina y el Caribe (CEPAL).

Wade, R. (1990): Governing the Market: Economic Theory and the Role of Government in East Asian Industrialization, Princeton, Princeton University Press.

Young, A. (1928): Increasing returns and economic progress, Economic Journal, vol. 38, $\mathrm{N}^{\circ}$ 4, Oxford, Reino Unido, Blackwell Publishing. 